\title{
Asymptotic stability of porous-elastic system with thermoelasticity of type III
}

Received: 2 June 2020 / Accepted: 18 November 2020 / Published online: 6 January 2021

(C) The Author(s) 2021

\begin{abstract}
In this work, we investigate a one-dimensional porous-elastic system with thermoelasticity of type III. We establish the well-posedness and the stability of the system for the cases of equal and nonequal speeds of wave propagation. At the end, we use some numerical approximations based on finite difference techniques to validate the theoretical results.
\end{abstract}

Mathematics Subject Classification $35 \mathrm{~B} 05 \cdot 35 \mathrm{~L} 05 \cdot 35 \mathrm{~L} 15 \cdot 35 \mathrm{~L} 70$

\section{Introduction}

In the recent decades, the study of problems related to elastic solids with voids has attracted the attention of many researchers due to the extensive practical applications of such materials in different fields, such as petroleum industry, foundation engineering, soil mechanics, power technology, biology, material science and so on. Elastic solids with voids are one of the simple extensions of the theory of the classical elasticity. It allows the treatment of porous solids in which the matrix material is elastic and the interstices are void of material.

In 1972, Godman and Cowin [12] proposed an extension of the classical elasticity theory to porous media. They introduced the concept of a continuum theory of granular materials with interstitial voids into the theory of elastic solids with voids. In addition to their usual elastic effects, these materials have a microstructure with the property that the mass at each point is obtained as the product of the mass density of the material matrix by the volume fraction. This latter idea was introduced by Nunziato and Cowin [22] when they developed a nonlinear theory of elastic materials with voids. We refer the reader to $[8,9,18,24]$ and the references therein for more details.

It is well known that the classical thermoelasticity using Fourier's Law of heat conduction, which states that the heat flux is proportional to the gradient of temperature $(q=-\delta \nabla \theta)$, leads to the physical paradox of infinite speed of heat propagation. In other words, any thermal disturbance at one point will be instantaneously transferred to the other parts of the body. This is practically unrealistic. To overcome this physical paradox but still keeping the essentials of heat conduction process, many theories have subsequently emerged. One of such theories was proposed by Green and Naghdi in 1990s [14-16]. They used an analogy between the concepts and equations of the purely thermal and the purely mechanical theories and arrived at three types of constitutive equations for heat flow in a stationary rigid solid labeled as type I, II, and III. Consequently, using

I. Lacheheb

Laboratoire de Mathématiques Appliquées, Université Kasdi Merbah, BP 511, 30000 Ouargla, Algeria

E-mail: lacheheb.ilyes@univ-ouargla.dz

S. A. Messaoudi $(\bowtie) \cdot$ M. Zahri

Department of Mathematics, University of Sharjah, Sharjah, United Arab Emirates

E-mail: smessaoudi@sharjah.ac.ae; mzahri@sharjah.ac.ae 
these constitutive equations, they obtained three models, called thermoelasticity of type I, thermoelasticity of type II, and thermoelasticity of type III. The linear version of the first one coincides with the classical theory based on Fourier's law, the second one is known as thermoelasticity without energy dissipation because the heat equation is not a dissipative process and the third one is the most general and it contains the former two as limit cases. For a further historical review on these models, we refer the reader to $[6,7,13-16]$. by:

The basic evolution equations for one-dimensional theories of porous materials with temperature are given

$$
\rho \omega_{t t}-T_{x}=0, \quad J \varphi_{t t}-H_{x}-G=0, \quad \alpha \theta_{t}+q_{x}+\beta \varphi_{t x}=0
$$

where $T$ is the stress tensor, $H$ is the equilibrated stress vector, $G$ is the equilibrated body force, and $q$ is the heat flux vector. The variables $\omega, \varphi$, and $\theta$ are the displacement of the solid elastic material, the volume fraction, and the difference temperature, respectively. The positive parameters $\rho, J$, and $\beta$ are the mass density, product of the mass density by the equilibrated inertia, and the coupling constant, respectively. Taking into account Green and Naghdi's theory, the constitutive equations are:

$$
\begin{aligned}
& T=\mu \omega_{x}+b \varphi, \quad H=\delta \varphi_{x}-\beta \theta \\
& G=-b \omega_{x}-\xi \varphi, \quad q=-\delta \Theta_{x}-k \Theta_{t x}
\end{aligned}
$$

where $\delta, k$ denote the thermal conductivity, $\Theta$ is the so-called thermal displacement whose time derivative is the empirical temperature $\theta$, that is, $\Theta_{t}=\theta$, and $\mu, \xi$ are constitutive constants which satisfy

$$
\mu>0, \quad \xi>0, \mu \xi>b^{2} .
$$

To keep the coupling, the constant $b$ must be different from zero. We substitute (1.2) into (1.1) to obtain the following system

$$
\begin{cases}\rho \omega_{t t}-\mu \omega_{x x}-b \varphi_{x}=0, & \text { in }(0,1) \times(0,+\infty) \\ J \varphi_{t t}-\delta \varphi_{x x}+b \omega_{x}+\xi \varphi+\beta \theta_{x}=0, & \text { in }(0,1) \times(0,+\infty) \\ \alpha \theta_{t}-\delta \Theta_{x x}+\beta \varphi_{t x}-k \Theta_{t x x}=0, & \text { in }(0,1) \times(0,+\infty) .\end{cases}
$$

For the asymptotic behaviors of the solutions for porous-elastic systems. Quintanilla [25] considered the one-dimensional porous dissipation elasticity:

$$
\begin{cases}\rho \omega_{t t}-\mu \omega_{x x}-b \varphi_{x}=0, & \text { in }(0, L) \times(0,+\infty) \\ J \varphi_{t t}-\delta \varphi_{x x}+b \omega_{x}+\xi \varphi+\tau \varphi_{t}=0, & \text { in }(0, L) \times(0,+\infty)\end{cases}
$$

with initial and boundary conditions. He used Hurtwitz theorem to prove that the damping through porousviscosity $\left(\tau \varphi_{t}\right)$ is not strong enough to obtain an exponential decay but only a slow (nonexponential) decay. However, Apalara [2,3] considered the same system and proved the exponential stability provided $\frac{\mu}{\rho}=\frac{\delta}{J}$. For various other damping mechanisms used and more results on porous elasticity, we refer the reader to [5,26-28] and the references therein. Recently, Apalara [4] considered the following porous-elastic system with microtemperature:

$$
\begin{cases}\rho \omega_{t t}-\mu \omega_{x x}-b \varphi_{x}=0, & \text { in }(0,1) \times(0,+\infty) \\ J \varphi_{t t}-\delta \varphi_{x x}+b \omega_{x}+\xi \varphi+\beta \theta_{x}=0, & \text { in }(0,1) \times(0,+\infty) \\ \alpha \theta_{t}-\kappa \theta_{x x}+\beta \varphi_{t x}+k \theta=0, & \text { in }(0,1) \times(0,+\infty)\end{cases}
$$

with Dirichlet-Neumann-Dirichlet boundary conditions. He showed that the unique dissipation given by microtemperatures is strong enough to produce exponential stability if and only if:

$$
\chi=\frac{\mu}{\rho}-\frac{\delta}{J}=0
$$

and showed that the system is polynomially stable if $\chi \neq 0$.

In the present work, we consider the system (1.4) which can be written as follows:

$$
\begin{cases}\rho \omega_{t t}-\mu \omega_{x x}-b \varphi_{x}=0, & \text { in }(0,1) \times(0,+\infty) \\ J \varphi_{t t}-\delta \varphi_{x x}+b \omega_{x}+\xi \varphi+\beta \theta_{x}=0, & \text { in }(0,1) \times(0,+\infty) \\ \alpha \theta_{t t}-\delta \theta_{x x}+\beta \varphi_{t t x}-k \theta_{t x x}=0, & \text { in }(0,1) \times(0,+\infty)\end{cases}
$$


with the following boundary conditions

$$
\omega(0, t)=\omega(1, t)=\varphi_{x}(0, t)=\varphi_{x}(1, t)=\theta(0, t)=\theta(1, t)=0, \quad \forall t \geq 0
$$

and initial conditions

$$
\begin{cases}\omega(x, 0)=\omega_{0}(x), \omega_{t}(x, 0)=\omega_{1}(x), & x \in(0,1) \\ \varphi(x, 0)=\varphi_{0}(x), \varphi_{t}(x, 0)=\varphi_{1}(x), & x \in(0,1) \\ \theta(x, 0)=\theta_{0}(x), \quad \theta_{t}(x, 0)=\theta_{1}(x), & x \in(0,1)\end{cases}
$$

We study the well-posedness and the asymptotic stability of (1.8)-(1.10). Using the semigroup theory, we prove the existence and uniqueness of the solution. We then exploit the energy method to obtain the exponential decay result for the case of equal wave speeds. When (1.7) does not hold, we prove a polynomial decay result.

The paper is organized as follows: in Sect. 2, we state the problem. In Sect. 3, we establish the wellposedness of the system. In Sect. 4, we show that the system is exponentially stable under condition (1.7). The polynomial stability when the wave-propagation speeds are different, is given in Sect. 5. In Sect. 6, we give some numerical illustrations.

\section{Statement of the problem}

To obtain the dissipative nature of System (1.8), we introduce the new variables: $u=\omega_{t}$ and $\phi=\varphi_{t}$. So, System (1.8) takes the form

$$
\begin{cases}\rho u_{t t}-\mu u_{x x}-b \phi_{x}=0, & \text { in }(0,1) \times(0,+\infty) \\ J \phi_{t t}-\delta \phi_{x x}+b u_{x}+\xi \phi+\beta \theta_{t x}=0, & \text { in }(0,1) \times(0,+\infty) \\ \alpha \theta_{t t}-\delta \theta_{x x}+\beta \phi_{t x}-k \theta_{t x x}=0, & \text { in }(0,1) \times(0,+\infty)\end{cases}
$$

with the following boundary conditions

$$
u(0, t)=u(1, t)=\phi_{x}(0, t)=\phi_{x}(1, t)=\theta(0, t)=\theta(1, t)=0, \quad \forall t \geq 0
$$

and initial conditions

$$
\begin{cases}u(x, 0)=u_{0}(x), \quad u_{t}(x, 0)=u_{1}(x), & x \in(0,1) \\ \phi(x, 0)=\phi_{0}(x), \quad \phi_{t}(x, 0)=\phi_{1}(x), & x \in(0,1) \\ \theta(x, 0)=\theta_{0}(x), \quad \theta_{t}(x, 0)=\theta_{1}(x), & x \in(0,1)\end{cases}
$$

Since the boundary conditions on $\phi$ are of Newmann type, we introduce some transformation that allows the use of Poincaré's inequality on $\phi$. From the second equation in (2.1) and the boundary conditions (2.2), it follows that

$$
\frac{\mathrm{d}^{2}}{\mathrm{~d} t^{2}} \int_{0}^{1} \phi(x, t) \mathrm{d} x+\frac{\xi}{J} \int_{0}^{1} \phi(x, t) \mathrm{d} x=0 .
$$

So, by solving (2.4) and using the initial data of $\phi$, we obtain

$$
\int_{0}^{1} \phi(x, t) \mathrm{d} x=\left(\int_{0}^{1} \phi_{0}(x) \mathrm{d} x\right) \cos \left(\sqrt{\frac{\xi}{J}} t\right)+\sqrt{\frac{J}{\xi}}\left(\int_{0}^{1} \phi_{1}(x) \mathrm{d} x\right) \sin \left(\sqrt{\frac{\xi}{J}} t\right) .
$$

Consequently, if we let

$$
\bar{\phi}(x, t)=\phi(x, t)-\left(\int_{0}^{1} \phi_{0}(x) \mathrm{d} x\right) \cos \left(\sqrt{\frac{\xi}{J}} t\right)-\sqrt{\frac{J}{\xi}}\left(\int_{0}^{1} \phi_{1}(x) \mathrm{d} x\right) \sin \left(\sqrt{\frac{\xi}{J}} t\right),
$$

we get

$$
\int_{0}^{1} \bar{\phi}(x, t) \mathrm{d} x=0, \forall t \geq 0
$$

which allows the use of Poincaré's inequality on $\bar{\phi}$. So, $(u, \bar{\phi}, \theta)$ satisfies (2.1), (2.2) and similar initial conditions (2.3). Therefore, we work with $(u, \bar{\phi}, \theta)$ but we write $(u, \phi, \theta)$ for simplicity. 


\section{The well-posedness of the problem}

In this section, we prove the existence, uniqueness and smoothness of solutions for the system (2.1)-(2.3) using the semigroup theory. Introducing the vector function $U=(u, v, \phi, \psi, \theta, q)^{T}$, where $v=u_{t}, \psi=\phi_{t}$ and $q=\theta_{t}$. System (2.1)-(2.3) can be written as

$$
\left\{\begin{array}{l}
U^{\prime}(t)=\mathcal{A} U(t), t>0 \\
U(0)=U_{0}
\end{array}\right.
$$

where $U_{0}=\left(u_{0}, u_{1}, \phi_{0}, \phi_{1}, \theta_{0}, \theta_{1}\right)^{\mathrm{T}}$ and the operator $\mathcal{A}$ is defined by

$$
\mathcal{A} U=\left(\begin{array}{l}
v \\
\frac{1}{\rho}\left(\mu u_{x x}+b \phi_{x}\right) \\
\psi \\
\frac{1}{J}\left(\delta \phi_{x x}-b u_{x}-\xi \phi-\beta q_{x}\right) \\
q \\
\frac{1}{\alpha}\left(\delta \theta_{x x}-\beta \psi_{x}+k q_{x x}\right)
\end{array}\right)
$$

We consider the energy space

$$
\mathcal{H}=H_{0}^{1}(0,1) \times L^{2}(0,1) \times H_{*}^{1}(0,1) \times L_{*}^{2}(0,1) \times H_{0}^{1}(0,1) \times L^{2}(0,1),
$$

where

$$
\begin{aligned}
& L_{*}^{2}(0,1)=\left\{u \in L^{2}(0,1) / \int_{0}^{1} u \mathrm{~d} x=0\right\} \\
& H_{*}^{1}(0,1)=\left\{u \in H^{1}(0,1) / \int_{0}^{1} u \mathrm{~d} x=0\right\}=H^{1}(0,1) \cap L_{*}^{2}(0,1) .
\end{aligned}
$$

$\mathcal{H}$ is a Hilbert space with respect to the following inner product

$$
\begin{aligned}
(U, \tilde{U})_{\mathcal{H}}:= & \rho \int_{0}^{1} v \tilde{v} \mathrm{~d} x+\xi \int_{0}^{1} \phi \tilde{\phi} \mathrm{d} x+J \int_{0}^{1} \psi \tilde{\psi} \mathrm{d} x+\alpha \int_{0}^{1} q \tilde{q} \mathrm{~d} x \\
& +\mu \int_{0}^{1} u_{x} \tilde{u}_{x} \mathrm{~d} x+\delta \int_{0}^{1}\left(\phi_{x} \tilde{\phi}_{x}+\theta_{x} \tilde{\theta}_{x}\right) \mathrm{d} x+b \int_{0}^{1}\left(u_{x} \tilde{\phi}+\phi \tilde{u}_{x}\right) \mathrm{d} x .
\end{aligned}
$$

Remark 3.1 Under the hypothesis $\mu \xi>b^{2}$, it is easy to see that (3.3) defines an inner product. In fact, from (3.3), we have

$$
\begin{aligned}
\|U\|_{\mathcal{H}}^{2}= & (U, U)_{\mathcal{H}}=\rho \int_{0}^{1} v^{2} \mathrm{~d} x+\left(\xi-\frac{b^{2}}{\mu}\right) \int_{0}^{1} \phi^{2} \mathrm{~d} x+J \int_{0}^{1} \psi^{2} \mathrm{~d} x+\alpha \int_{0}^{1} q^{2} \mathrm{~d} x \\
& +\mu \int_{0}^{1}\left(u_{x}+\frac{b}{\mu} \phi\right)^{2} \mathrm{~d} x+\delta \int_{0}^{1}\left(\phi_{x}^{2}+\theta_{x}^{2}\right) \mathrm{d} x .
\end{aligned}
$$

Hence, since $\mu \xi>b^{2}$ we conclude that $(U, \tilde{U})_{\mathcal{H}}$ defines an inner product on $\mathcal{H}$ and the associated norm $\|\cdot\|_{\mathcal{H}}$ is equivalent to the usual one.

The domain of $\mathcal{A}$ is given by

$$
\begin{aligned}
& D(\mathcal{A})=\left\{U \in \mathcal{H}: u \in H^{2}(0,1) \cap H_{0}^{1}(0,1), \quad v \in H_{0}^{1}(0,1), \quad \phi \in H_{*}^{2}(0,1) \cap H_{*}^{1}(0,1),\right. \\
& \left.\psi \in H_{*}^{1}(0,1), \quad q \in H_{0}^{1}(0,1), \quad(\delta \theta+k q) \in H^{2}(0,1)\right\},
\end{aligned}
$$

where

$$
H_{*}^{2}(0,1)=\left\{u \in H^{2}(0,1): u_{x}(0)=u_{x}(1)=0\right\} .
$$

We have the following well-posedness result: 
Theorem 3.2 Let $U_{0} \in \mathcal{H}$. Then, there exists a unique solution $U \in C\left(\mathbb{R}_{+}, \mathcal{H}\right)$ of problem (2.1)-(2.3). Moreover, if $U_{0} \in D(\mathcal{A})$, then $U \in C\left(\mathbb{R}_{+}, D(\mathcal{A})\right) \cap C^{1}\left(\mathbb{R}_{+}, \mathcal{H}\right)$.

Proof The result follows from Lumer-Phillips theorem (see [19,23]) provided we prove that $\mathcal{A}$ is a maximal dissipative operator, that is $\mathcal{A}$ is dissipative and that $(I-\mathcal{A})$ is surjective. Thus, for any $U \in D(\mathcal{A})$, we have

$$
\begin{aligned}
(\mathcal{A} U, U) \mathcal{H}= & \mu \int_{0}^{1} u_{x x} v \mathrm{~d} x+b \int_{0}^{1} \phi_{x} v \mathrm{~d} x+\xi \int_{0}^{1} \psi \phi \mathrm{d} x+\delta \int_{0}^{1} \phi_{x x} \psi \mathrm{d} x-b \int_{0}^{1} u_{x} \psi \mathrm{d} x \\
& -\xi \int_{0}^{1} \phi \psi \mathrm{d} x-\beta \int_{0}^{1} q_{x} \psi \mathrm{d} x+\delta \int_{0}^{1} \theta_{x x} q \mathrm{~d} x-\beta \int_{0}^{1} \psi_{x} q \mathrm{~d} x+k \int_{0}^{1} q_{x x} q \mathrm{~d} x \\
& +\mu \int_{0}^{1} u_{x} v_{x} \mathrm{~d} x+\delta \int_{0}^{1} \psi_{x} \phi_{x} \mathrm{~d} x+\delta \int_{0}^{1} \theta_{x} q_{x} \mathrm{~d} x+b \int_{0}^{1} v_{x} \phi \mathrm{d} x+b \int_{0}^{1} \psi u_{x} \mathrm{~d} x \\
= & -k \int_{0}^{1} q_{x}^{2} \mathrm{~d} x \leq 0 .
\end{aligned}
$$

So, $\mathcal{A}$ is dissipative. Next, we prove that the operator $(I-\mathcal{A})$ is surjective.

Let $F=\left(f^{1}, f^{2}, f^{3}, f^{4}, f^{5}, f^{6}\right)^{\mathrm{T}} \in \mathcal{H}$, we prove that there exists a unique $U \in D(\mathcal{A})$ satisfying

$$
(I-\mathcal{A}) U=F
$$

That is,

$$
\left\{\begin{array}{l}
u-v=f^{1} \\
\rho v-\mu u_{x x}-b \phi_{x}=\rho f^{2} \\
\phi-\psi=f^{3} \\
J \psi-\delta \phi_{x x}+b u_{x}+\xi \phi+\beta q_{x}=J f^{4} \\
\theta-q=f^{5} \\
\alpha q-\delta \theta_{x x}+\beta \psi_{x}-k q_{x x}=\alpha f^{6} .
\end{array}\right.
$$

Using Eqs. $(3.5)_{1},(3.5)_{3},(3.5)_{5}$ in $(3.5)_{2},(3.5)_{4},(3.5)_{6}$, respectively, we obtain

$$
\left\{\begin{array}{l}
\rho u-\mu u_{x x}-b \phi_{x}=\rho\left(f^{2}+f^{1}\right) \\
J \phi-\delta \phi_{x x}+b u_{x}+\xi \phi+\beta \theta_{x}=J\left(f^{4}+f^{3}\right)+\beta f_{x}^{5} \\
\alpha \theta-(\delta+k) \theta_{x x}+\beta \phi_{x}=\alpha\left(f^{6}+f^{5}\right)+\beta f_{x}^{3}-k f_{x x}^{5} .
\end{array}\right.
$$

To solve (3.6), we consider the following variational formulation

$$
B((u, \phi, \theta),(\tilde{u}, \tilde{\phi}, \tilde{\theta}))=L((\tilde{u}, \tilde{\phi}, \tilde{\theta})), \quad \forall(\tilde{u}, \tilde{\phi}, \tilde{\theta}) \in \mathcal{W}
$$

where $\mathcal{W}=H_{0}^{1}(0,1) \times H_{*}^{1}(0,1) \times H_{0}^{1}(0,1), B: \mathcal{W} \times \mathcal{W} \longrightarrow \mathbb{R}$ is the bilinear form defined by

$$
\begin{aligned}
& \rho \int_{0}^{1} u \tilde{u} \mathrm{~d} x+\mu \int_{0}^{1} u_{x} \tilde{u}_{x} \mathrm{~d} x-b \int_{0}^{1} \phi_{x} \tilde{u} \mathrm{~d} x+(J+\xi) \int_{0}^{1} \phi \tilde{\phi} \mathrm{d} x+\delta \int_{0}^{1} \phi_{x} \tilde{\phi}_{x} \mathrm{~d} x+b \int_{0}^{1} u_{x} \tilde{\phi} \mathrm{d} x \\
& \quad+\beta \int_{0}^{1}\left(\theta_{x} \tilde{\phi}+\phi_{x} \tilde{\theta}\right) \mathrm{d} x+\alpha \int_{0}^{1} \theta \tilde{\theta} \mathrm{d} x+(\delta+k) \int_{0}^{1} \theta_{x} \tilde{\theta}_{x} \mathrm{~d} x
\end{aligned}
$$

and $L: \mathcal{W} \longrightarrow \mathbb{R}$ is the linear form given by

$$
\rho \int_{0}^{1}\left(f^{2}+f^{1}\right) \tilde{u} \mathrm{~d} x+\int_{0}^{1}\left(J\left(f^{4}+f^{3}\right)+\beta f_{x}^{5}\right) \tilde{\phi} \mathrm{d} x+\int_{0}^{1}\left(\alpha\left(f^{6}+f^{5}\right)+\beta f_{x}^{3}\right) \tilde{\theta} \mathrm{d} x+k \int_{0}^{1} f_{x}^{5} \tilde{\theta}_{x} \mathrm{~d} x .
$$

It is clear that $\mathcal{W}$ is a Hilbert space with the usual norm and we can easily show, using Cauchy-Schwarz inequality, that $B$ and $L$ are continuous. On the other hand, using Young's inequality and the fact $\mu \xi>b^{2}$, we have 


$$
\begin{aligned}
B((u, \phi, \theta),(u, \phi, \theta)) & \geq \rho\|u\|^{2}+\left(\mu-\frac{b^{2}}{\xi}\right)\left\|u_{x}\right\|^{2}+J\|\phi\|^{2}+\delta\left\|\phi_{x}\right\|^{2}+\alpha\|\theta\|^{2}+(k+\delta)\left\|\theta_{x}\right\|^{2} \\
& \geq c\|(u, \phi, \theta)\|_{\mathcal{W}}^{2},
\end{aligned}
$$

for some $c>0$. Hence, $B$ is coercive. Consequently, Lax-Milgram lemma guarantees the existence of a unique $(u, \phi, \theta)$ in $\mathcal{W}$ satisfying (3.7). Using (3.5), we have

$$
v=u-f^{1} \in H_{0}^{1}, \psi=\phi-f^{3} \in H_{*}^{1}, q=\theta-f^{5} \in H_{0}^{1} .
$$

- If we take $(\tilde{\phi}, \tilde{\theta})=(0,0)$ in $(3.7)$, we get

$$
\mu \int_{0}^{1} u_{x} \tilde{u}_{x} \mathrm{~d} x=\int_{0}^{1}\left[\rho\left(f^{1}+f^{2}-u\right)+b \phi_{x}\right] \tilde{u} \mathrm{~d} x, \forall \tilde{u} \in H_{0}^{1}(0,1) .
$$

Thus, the elliptic regularity theory implies that

$$
u \in H^{2}(0,1)
$$

and, moreover, we obtain

$$
\rho u-\mu u_{x x}-b \phi_{x}=\rho\left(f^{1}+f^{2}\right) .
$$

Since $f^{1}=u-v$, then

$$
\rho v-\mu u_{x x}-b \phi_{x}=\rho f^{2}
$$

which solves $(3.5)_{2}$.

- If $(\tilde{u}, \tilde{\theta})=(0,0)$ in $(3.7)$, then we have

$$
\delta \int_{0}^{1} \phi_{x} \tilde{\phi}_{x} \mathrm{~d} x=\int_{0}^{1}\left[J\left(f^{3}+f^{4}\right)+\beta f_{x}^{5}-(J+\xi) \phi-b u_{x}-\beta \theta_{x}\right] \tilde{\phi} \mathrm{d} x, \forall \tilde{\phi} \in H_{*}^{1}(0,1) .
$$

Here, we can't use the regularity theorem, because $\tilde{\phi} \in H_{*}^{1}(0,1)$. So, we take $\tilde{\Psi} \in H_{0}^{1}(0,1)$ and set

$$
\tilde{\phi}(x)=\tilde{\Psi}(x)-\int_{0}^{1} \tilde{\Psi}(x) \mathrm{d} x .
$$

It is clear that $\tilde{\phi} \in H_{*}^{1}(0,1)$. Then, a substitution in (3.8) leads to

$$
\delta \int_{0}^{1} \phi_{x} \tilde{\Psi}_{x} \mathrm{~d} x=\int_{0}^{1} r \tilde{\Psi} \mathrm{d} x, \quad \forall \tilde{\Psi} \in H_{0}^{1}(0,1)
$$

where

$$
r=J\left(f^{3}+f^{4}\right)+\beta f_{x}^{5}-(J+\xi) \phi-b u_{x}-\beta \theta_{x} \in L_{*}^{2}(0,1) .
$$

So

$$
\phi \in H^{2}(0,1)
$$

and

$$
-\delta \phi_{x x}=J\left(f^{3}+f^{4}\right)+\beta f_{x}^{5}-(J+\xi) \phi-b u_{x}-\beta \theta_{x} .
$$

We use $f^{3}=\phi-\psi$ and $f^{5}=\theta-q$ to obtain

$$
J \psi-\delta \phi_{x x}+b u_{x}+\xi \phi+\beta q_{x}=J f^{4} .
$$

This gives (3.5) $)_{4}$. Since $-\delta \phi_{x x}=r(x)$, then

$$
-\delta \int_{0}^{1} \phi_{x x} \Phi \mathrm{d} x=\int_{0}^{1} r \Phi \mathrm{d} x, \quad \forall \Phi \in H^{1}(0,1) .
$$


Namely,

$$
-\left.\delta \phi_{x} \Phi\right|_{0} ^{1}+\delta \int_{0}^{1} \phi_{x} \Phi_{x} \mathrm{~d} x=\int_{0}^{1} r \Phi \mathrm{d} x, \quad \forall \Phi \in H^{1}(0,1) .
$$

Since $H_{*}^{1} \subset H^{1}$. Then, we have

$$
-\left.\delta \phi_{x} \tilde{\phi}\right|_{0} ^{1}+\delta \int_{0}^{1} \phi_{x} \tilde{\phi}_{x} \mathrm{~d} x=\int_{0}^{1} r \tilde{\phi} \mathrm{d} x, \quad \forall \tilde{\phi} \in H_{*}^{1}(0,1),
$$

and the other hand, we have (3.8). Thus

$$
\phi_{x}(1) \tilde{\phi}(1)-\phi_{x}(0) \tilde{\phi}(0)=0, \quad \forall \tilde{\phi} \in H_{*}^{1}(0,1) .
$$

Since $\tilde{\phi} \in H_{*}^{1}$ is arbitrary. Then,

$$
\phi_{x}(1)=\phi_{x}(0)=0 \text {, }
$$

and, hence,

$$
\phi \in H_{*}^{2}(0,1) .
$$

- If $(\tilde{u}, \tilde{\phi})=(0,0)$ in (3.7) we get, for any $\tilde{\theta} \in H_{0}^{1}(0,1)$,

$$
\alpha \int_{0}^{1} \theta \tilde{\theta} \mathrm{d} x+(\delta+k) \int_{0}^{1} \theta_{x} \tilde{\theta}_{x} \mathrm{~d} x+\beta \int_{0}^{1} \phi_{x} \tilde{\theta} \mathrm{d} x-k \int_{0}^{1} f_{x}^{5} \tilde{\theta}_{x} \mathrm{~d} x=\int_{0}^{1}\left[\alpha\left(f^{6}+f^{5}\right)+\beta f_{x}^{3}\right] \tilde{\theta} \mathrm{d} x
$$

This, in turns, yields

$$
\int_{0}^{1}\left[(\delta+k) \theta_{x}-k f_{x}^{5}\right] \tilde{\theta}_{x} \mathrm{~d} x=\int_{0}^{1} R \tilde{\theta} \mathrm{d} x, \quad \forall \tilde{\theta} \in H_{0}^{1}(0,1)
$$

where

$$
R=\alpha\left(f^{6}+f^{5}\right)+\beta f_{x}^{3}-\alpha \theta-\beta \phi_{x} .
$$

Then,

$$
\left[(\delta+k) \theta-k f^{5}\right] \in H^{2}(0,1)
$$

Since $f^{5}=\theta-q$, then $(\delta \theta+k q) \in H^{2}(0,1)$ and we have

$$
\alpha q-\delta \theta_{x x}+\beta \psi_{x}-k q_{x x}=\alpha f^{6}
$$

which solves $(3.5)_{6}$.

Hence, there exists a unique $U \in D(\mathcal{A})$ satisfies (3.4). Finally, using Lumer-Phillips theorem we deduce that $\mathcal{A}$ is an infinitesimal generator of a contraction semigroup in $\mathcal{H}$ and this complete the proof. 


\section{Exponential stability}

In this section, we use the energy method to prove that system (2.1)-(2.3) is exponentially stable in the case of equal wave-speed propagation (1.7). To achieve this goal, we first establish some technical lemmas needed in the proof of exponential stability result. We also use $c$ to be a positive generic constant.

Lemma 4.1 Let $(u, \phi, \theta)$ be the solution of (2.1)-(2.3). Then the energy functional $E$, defined by

$$
E(t)=\frac{1}{2} \int_{0}^{1}\left[\rho u_{t}^{2}+J \phi_{t}^{2}+\alpha \theta_{t}^{2}+\mu u_{x}^{2}+\delta \phi_{x}^{2} \mathrm{~d} x+\delta \theta_{x}^{2}+2 b u_{x} \phi+\xi \phi^{2}\right] \mathrm{d} x
$$

satisfies

$$
E^{\prime}(t)=-k \int_{0}^{1} \theta_{t x}^{2} \mathrm{~d} x \leq 0 .
$$

Proof Multiplying (2.1) by $u_{t}, \phi_{t}$ and $\theta_{t}$ respectively, integrating over $(0,1)$ and using integration by parts and the boundary conditions, we obtain

$$
\frac{\rho}{2} \frac{\mathrm{d}}{\mathrm{d} t} \int_{0}^{1} u_{t}^{2} \mathrm{~d} x+\frac{\mu}{2} \frac{\mathrm{d}}{\mathrm{d} t} \int_{0}^{1} u_{x}^{2} \mathrm{~d} x+b \frac{\mathrm{d}}{\mathrm{d} t} \int_{0}^{1} \phi u_{x} \mathrm{~d} x-b \int_{0}^{1} \phi_{t} u_{x} \mathrm{~d} x=0 .
$$

The second equation

$$
\frac{J}{2} \frac{\mathrm{d}}{\mathrm{d} t} \int_{0}^{1} \phi_{t}^{2} \mathrm{~d} x+\frac{\delta}{2} \frac{\mathrm{d}}{\mathrm{d} t} \int_{0}^{1} \phi_{x}^{2} \mathrm{~d} x+b \int_{0}^{1} u_{x} \phi_{t} \mathrm{~d} x+\frac{\xi}{2} \frac{\mathrm{d}}{\mathrm{d} t} \int_{0}^{1} \phi^{2} \mathrm{~d} x+\beta \int_{0}^{1} \theta_{t x} \phi_{t} \mathrm{~d} x=0 .
$$

The third equation

$$
\frac{\alpha}{2} \frac{\mathrm{d}}{\mathrm{d} t} \int_{0}^{1} \theta_{t}^{2} \mathrm{~d} x+\frac{\delta}{2} \frac{\mathrm{d}}{\mathrm{d} t} \int_{0}^{1} \theta_{x}^{2} \mathrm{~d} x-\beta \int_{0}^{1} \phi_{t} \theta_{t x} \mathrm{~d} x+k \int_{0}^{1} \theta_{t x}^{2} \mathrm{~d} x=0 .
$$

Adding up the above identities we arrive at

$$
\frac{1}{2} \frac{\mathrm{d}}{\mathrm{d} t} \int_{0}^{1}\left[\rho u_{t}^{2}+J \phi_{t}^{2}+\alpha \theta_{t}^{2}+\mu u_{x}^{2}+\delta \phi_{x}^{2}+\delta \theta_{x}^{2}+2 b \phi u_{x}+\xi \phi^{2}\right] \mathrm{d} x=-k \int_{0}^{1} \theta_{t x}^{2} \mathrm{~d} x .
$$

This is exactly (4.2).

Lemma 4.2 Let $(u, \phi, \theta)$ be the solution of (2.1)-(2.3). Then the functional

$$
F_{1}(t):=J \int_{0}^{1} \phi \phi_{t} \mathrm{~d} x-\frac{\rho b}{\mu} \int_{0}^{1} u_{t}\left(\int_{0}^{x} \phi(y) \mathrm{d} y\right) \mathrm{d} x
$$

satisfies, for any $\varepsilon_{1}>0$, the estimate

$$
F_{1}^{\prime}(t) \leq-\frac{\delta}{2} \int_{0}^{1} \phi_{x}^{2} \mathrm{~d} x-\left(\xi-\frac{b^{2}}{\mu}\right) \int_{0}^{1} \phi^{2} \mathrm{~d} x+\varepsilon_{1} \int_{0}^{1} u_{t}^{2} \mathrm{~d} x+\frac{\beta^{2}}{2 \delta} \int_{0}^{1} \theta_{t}^{2} \mathrm{~d} x+\left(J+\frac{c}{\varepsilon_{1}}\right) \int_{0}^{1} \phi_{t}^{2} \mathrm{~d} x .
$$

Proof By taking the derivative of $F_{1}$, using (2.1) and integrating by parts, we get

$$
\begin{aligned}
F_{1}^{\prime}(t)= & J \int_{0}^{1} \phi_{t}^{2} \mathrm{~d} x+J \int_{0}^{1} \phi \phi_{t t} \mathrm{~d} x-\frac{\rho b}{\mu} \int_{0}^{1} u_{t t}\left(\int_{0}^{x} \phi \mathrm{d} y\right) \mathrm{d} x-\frac{\rho b}{\mu} \int_{0}^{1} u_{t}\left(\int_{0}^{x} \phi_{t} \mathrm{~d} y\right) \mathrm{d} x \\
= & J \int_{0}^{1} \phi_{t}^{2} \mathrm{~d} x+\delta \int_{0}^{1} \phi \phi_{x x} \mathrm{~d} x-b \int_{0}^{1} \phi u_{x} \mathrm{~d} x-\xi \int_{0}^{1} \phi^{2} \mathrm{~d} x-\beta \int_{0}^{1} \phi \theta_{t x} \mathrm{~d} x \\
& -b \int_{0}^{1} u_{x x}\left(\int_{0}^{x} \phi \mathrm{d} y\right) \mathrm{d} x-\frac{b^{2}}{\mu} \int_{0}^{1} \phi_{x}\left(\int_{0}^{x} \phi(y) \mathrm{d} y\right) \mathrm{d} x-\frac{b \rho}{\mu} \int_{0}^{1} u_{t}\left(\int_{0}^{x} \phi_{t}(y) \mathrm{d} y\right) \mathrm{d} x .
\end{aligned}
$$


We use integration by parts and $\int_{0}^{1} \phi \mathrm{d} x=0$ to obtain

$$
\int_{0}^{1} u_{x x}\left(\int_{0}^{x} \phi(y) \mathrm{d} y\right) \mathrm{d} x=-\int_{0}^{1} u_{x} \phi \mathrm{d} x
$$

and

$$
\int_{0}^{1} \phi_{x}\left(\int_{0}^{x} \phi(y) \mathrm{d} y\right) \mathrm{d} x=-\int_{0}^{1} \phi^{2} \mathrm{~d} x
$$

So,

$$
\begin{aligned}
F_{1}^{\prime}(t)= & J \int_{0}^{1} \phi_{t}^{2} \mathrm{~d} x-\delta \int_{0}^{1} \phi_{x}^{2} \mathrm{~d} x-b \int_{0}^{1} \phi u_{x} \mathrm{~d} x-\xi \int_{0}^{1} \phi^{2} \mathrm{~d} x+\beta \int_{0}^{1} \phi_{x} \theta_{t} \mathrm{~d} x+b \int_{0}^{1} u_{x} \phi \mathrm{d} x \\
& +\frac{b^{2}}{\mu} \int_{0}^{1} \phi^{2} \mathrm{~d} x-\frac{b \rho}{\mu} \int_{0}^{1} u_{t}\left(\int_{0}^{x} \phi_{t}(y) \mathrm{d} y\right) \mathrm{d} x .
\end{aligned}
$$

Using Young's and Cauchy-Schwarz inequalities, we have,

$$
\beta \int_{0}^{1} \phi_{x} \theta_{t} \mathrm{~d} x \leq \frac{\delta}{2} \int_{0}^{1} \phi_{x}^{2} \mathrm{~d} x+\frac{\beta^{2}}{2 \delta} \int_{0}^{1} \theta_{t}^{2} \mathrm{~d} x
$$

and, for any $\varepsilon_{1}>0$,

$$
\begin{aligned}
-\frac{b \rho}{\mu} \int_{0}^{1} u_{t}\left(\int_{0}^{x} \phi_{t}(y) \mathrm{d} y\right) \mathrm{d} x & \leq \varepsilon_{1} \int_{0}^{1} u_{t}^{2} \mathrm{~d} x+\frac{c}{\varepsilon_{1}} \int_{0}^{1}\left(\int_{0}^{x} \phi_{t}(y) \mathrm{d} y\right)^{2} \mathrm{~d} x \\
& \leq \varepsilon_{1} \int_{0}^{1} u_{t}^{2} \mathrm{~d} x+\frac{c}{\varepsilon_{1}} \int_{0}^{1} \phi_{t}^{2} \mathrm{~d} x .
\end{aligned}
$$

Then, by substituting the above inequalities into (4.7), we get

$$
\begin{aligned}
F_{1}^{\prime}(t) \leq & -\frac{\delta}{2} \int_{0}^{1} \phi_{x}^{2} \mathrm{~d} x-\left(\xi-\frac{b^{2}}{\mu}\right) \int_{0}^{1} \phi^{2} \mathrm{~d} x+\varepsilon_{1} \int_{0}^{1} u_{t}^{2} \mathrm{~d} x+\frac{\beta^{2}}{2 \delta} \int_{0}^{1} \theta_{t}^{2} \mathrm{~d} x \\
& +\left(J+\frac{c}{\varepsilon_{1}}\right) \int_{0}^{1} \phi_{t}^{2} \mathrm{~d} x .
\end{aligned}
$$

Lemma 4.3 Let $(u, \phi, \theta)$ be the solution of (2.1)-(2.3). Then the functional

$$
F_{2}(t):=-\alpha \int_{0}^{1} \theta_{t}\left(\int_{0}^{x} \phi_{t}(y) \mathrm{d} y\right) \mathrm{d} x
$$

satisfies, for any $\varepsilon_{2}>0$, the estimate

$$
F_{2}^{\prime}(t) \leq-\frac{\beta}{2} \int_{0}^{1} \phi_{t}^{2} \mathrm{~d} x+c \varepsilon_{2} \int_{0}^{1}\left(\phi_{x}^{2}+u_{x}^{2}\right) \mathrm{d} x+c\left(1+\frac{1}{\varepsilon_{2}}\right) \int_{0}^{1} \theta_{t x}^{2} \mathrm{~d} x+\frac{\delta^{2}}{\beta} \int_{0}^{1} \theta_{x}^{2} \mathrm{~d} x .
$$

Proof The differentiation of $F_{2}$, using (2.1), integration by parts, and the boundary conditions (2.2), gives

$$
\begin{aligned}
F_{2}^{\prime}(t)= & -\alpha \int_{0}^{1} \theta_{t t}\left(\int_{0}^{x} \phi_{t}(y) \mathrm{d} y\right) \mathrm{d} x-\alpha \int_{0}^{1} \theta_{t} \int_{0}^{x} \phi_{t t}(y) \mathrm{d} y \mathrm{~d} x \\
= & -\delta \int_{0}^{1} \theta_{x x} \int_{0}^{x} \phi_{t}(y) \mathrm{d} y \mathrm{~d} x+\beta \int_{0}^{1} \phi_{t x}\left(\int_{0}^{x} \phi_{t}(y) \mathrm{d} y\right) \mathrm{d} x \\
& -k \int_{0}^{1} \theta_{t x x}\left(\int_{0}^{x} \phi_{t}(y) \mathrm{d} y\right) \mathrm{d} x-\alpha \int_{0}^{1} \theta_{t}\left(\int_{0}^{x} \phi_{t t}(y) \mathrm{d} y\right) \mathrm{d} x . \\
= & \delta \int_{0}^{1} \theta_{x} \phi_{t} \mathrm{~d} x-\beta \int_{0}^{1} \phi_{t}^{2} \mathrm{~d} x+k \int_{0}^{1} \theta_{t x} \phi_{t} \mathrm{~d} x-\alpha \int_{0}^{1} \theta_{t} \int_{0}^{x} \phi_{t t}(y) \mathrm{d} y \mathrm{~d} x .
\end{aligned}
$$


Now, we estimate the terms in the right-hand side of the above identity. Using Young's and Cauchy-Schwarz inequalities, (2.1), and calculations as in (4.8), we find,

$$
\begin{aligned}
& \delta \int_{0}^{1} \theta_{x} \phi_{t} \mathrm{~d} x \leq \frac{\beta}{4} \int_{0}^{1} \phi_{t}^{2} \mathrm{~d} x+\frac{\delta^{2}}{\beta} \int_{0}^{1} \theta_{x}^{2} \mathrm{~d} x . \\
& k \int_{0}^{1} \theta_{t x} \phi_{t} \mathrm{~d} x \leq \frac{\beta}{4} \int_{0}^{1} \phi_{t}^{2} \mathrm{~d} x+\frac{k^{2}}{\beta} \int_{0}^{1} \theta_{t x}^{2} \mathrm{~d} x .
\end{aligned}
$$

and, for any $\varepsilon_{2}>0$,

$$
\begin{aligned}
-\alpha \int_{0}^{1} \theta_{t}\left(\int_{0}^{x} \phi_{t t}(y) \mathrm{d} y\right) \mathrm{d} x= & -\alpha \int_{0}^{1} \theta_{t}\left(\int_{0}^{x}\left(\frac{\delta}{J} \phi_{y y}-\frac{b}{J} u_{y}-\frac{\xi}{J} \phi-\frac{\beta}{J} \theta_{t y}\right) \mathrm{d} y\right) \mathrm{d} x \\
= & -\frac{\alpha \delta}{J} \int_{0}^{1} \theta_{t} \phi_{x} \mathrm{~d} x+\frac{\alpha b}{J} \int_{0}^{1} \theta_{t} u \mathrm{~d} x+\frac{\alpha \xi}{J} \int_{0}^{1} \theta_{t}\left(\int_{0}^{x} \phi \mathrm{d} y\right) \mathrm{d} x+\frac{\alpha \beta}{J} \int_{0}^{1} \theta_{t}^{2} \mathrm{~d} x \\
\leq & \varepsilon_{2} \int_{0}^{1} \phi_{x}^{2} \mathrm{~d} x+\frac{c}{\varepsilon_{2}} \int_{0}^{1} \theta_{t}^{2} \mathrm{~d} x+\varepsilon_{2} \int_{0}^{1} u^{2} \mathrm{~d} x+\frac{c}{\varepsilon_{2}} \int_{0}^{1} \theta_{t}^{2} \mathrm{~d} x \\
& +\varepsilon_{2} \int_{0}^{1} \phi^{2} \mathrm{~d} x+\frac{c}{\varepsilon_{2}} \int_{0}^{1} \theta_{t}^{2} \mathrm{~d} x+\frac{\alpha \beta}{J} \int_{0}^{1} \theta_{t}^{2} \mathrm{~d} x .
\end{aligned}
$$

So, by Poincaré's inequality and the above estimate, we arrive at

$$
\begin{aligned}
F_{2}^{\prime}(t) \leq & \frac{-\beta}{2} \int_{0}^{1} \phi_{t}^{2} \mathrm{~d} x+\left(\frac{\alpha \beta}{J}+\frac{c}{\varepsilon_{2}}\right) \int_{0}^{1} \theta_{t}^{2} \mathrm{~d} x+\varepsilon_{2} \int_{0}^{1} \phi^{2} \mathrm{~d} x+\varepsilon_{2} \int_{0}^{1} \phi_{x}^{2} \mathrm{~d} x+\varepsilon_{2} \int_{0}^{1} u^{2} \mathrm{~d} x \\
& +\frac{k^{2}}{\beta} \int_{0}^{1} \theta_{t x}^{2} \mathrm{~d} x+\frac{\delta^{2}}{\beta} \int_{0}^{1} \theta_{x}^{2} \mathrm{~d} x \\
\leq & \frac{-\beta}{2} \int_{0}^{1} \phi_{t}^{2} \mathrm{~d} x+c\left(1+\frac{1}{\varepsilon_{2}}\right) \int_{0}^{1} \theta_{t x}^{2} \mathrm{~d} x+\varepsilon_{2} c \int_{0}^{1}\left(\phi_{x}^{2}+u_{x}^{2}\right) \mathrm{d} x+\frac{\delta^{2}}{\beta} \int_{0}^{1} \theta_{x}^{2} \mathrm{~d} x .
\end{aligned}
$$

Lemma 4.4 Let $(u, \phi, \theta)$ be the solution of (2.1)-(2.3). Then the functional

$$
F_{3}(t):=\frac{\mu}{\rho} \int_{0}^{1} \phi_{t} u_{x} \mathrm{~d} x+\frac{\delta}{J} \int_{0}^{1} \phi_{x} u_{t} \mathrm{~d} x
$$

satisfies, for some positive constant $m_{0}$, the estimate

$$
F_{3}^{\prime}(t) \leq-m_{0} \int_{0}^{1} u_{x}^{2} \mathrm{~d} x+c \int_{0}^{1} \phi^{2} \mathrm{~d} x+c \int_{0}^{1} \theta_{t x}^{2} \mathrm{~d} x+\frac{\delta b}{\rho J} \int_{0}^{1} \phi_{x}^{2} \mathrm{~d} x .
$$

Proof Direct computations, exploiting $\chi=0(\chi=0$ defined by (2.7)) and using (2.1) and integration by parts, yield

$$
\begin{aligned}
F_{3}^{\prime}(t)= & \frac{\mu}{\rho} \int_{0}^{1} \phi_{t t} u_{x} \mathrm{~d} x+\frac{\mu}{\rho} \int_{0}^{1} \phi_{t} u_{t x} \mathrm{~d} x+\frac{\delta}{J} \int_{0}^{1} \phi_{t x} u_{t} \mathrm{~d} x+\frac{\delta}{J} \int_{0}^{1} \phi_{x} u_{t t} \mathrm{~d} x \\
= & \frac{\mu \delta}{\rho J} \int_{0}^{1} \phi_{x x} u_{x} \mathrm{~d} x-\frac{b \mu}{\rho J} \int_{0}^{1} u_{x}^{2} \mathrm{~d} x-\frac{\mu \xi}{\rho J} \int_{0}^{1} \phi u_{x} \mathrm{~d} x-\frac{\beta \mu}{\rho J} \int_{0}^{1} \theta_{t x} u_{x} \mathrm{~d} x-\chi \int_{0}^{1} \phi_{t x} u_{t} \mathrm{~d} x \\
& +\frac{\delta \mu}{J \rho} \int_{0}^{1} \phi_{x} u_{x x} \mathrm{~d} x+\frac{\delta b}{\rho J} \int_{0}^{1} \phi_{x}^{2} \mathrm{~d} x \\
= & \frac{-b \mu}{\rho J} \int_{0}^{1} u_{x}^{2} \mathrm{~d} x-\frac{\mu \xi}{\rho J} \int_{0}^{1} \phi u_{x} \mathrm{~d} x-\frac{\beta \mu}{\rho J} \int_{0}^{1} \theta_{t x} u_{x} \mathrm{~d} x+\frac{\delta b}{\rho J} \int_{0}^{1} \phi_{x}^{2} \mathrm{~d} x-\chi \int_{0}^{1} \phi_{t x} u_{t} \\
= & \frac{-b \mu}{\rho J} \int_{0}^{1} u_{x}^{2} \mathrm{~d} x-\frac{\mu \xi}{\rho J} \int_{0}^{1} \phi u_{x} \mathrm{~d} x-\frac{\beta \mu}{\rho J} \int_{0}^{1} \theta_{t x} u_{x} \mathrm{~d} x+\frac{\delta b}{\rho J} \int_{0}^{1} \phi_{x}^{2} \mathrm{~d} x .
\end{aligned}
$$


Arab. J. Math. (2021) 10:137-155

147

Using Young's inequality, we get, for any $\varepsilon_{3}>0$,

$$
\begin{aligned}
F_{3}^{\prime}(t) \leq & \frac{-b \mu}{\rho J} \int_{0}^{1} u_{x}^{2} \mathrm{~d} x+\frac{\varepsilon_{3}}{2} \int_{0}^{1} u_{x}^{2} \mathrm{~d} x+\frac{c}{\varepsilon_{3}} \int_{0}^{1} \phi^{2} \mathrm{~d} x \\
& +\frac{\varepsilon_{3}}{2} \int_{0}^{1} u_{x}^{2} \mathrm{~d} x+\frac{c}{\varepsilon_{3}} \int_{0}^{1} \theta_{t x}^{2} \mathrm{~d} x+\frac{\delta b}{\rho J} \int_{0}^{1} \phi_{x}^{2} \mathrm{~d} x \\
\leq & -\left(\frac{b \mu}{\rho J}-\varepsilon_{3}\right) \int_{0}^{1} u_{x}^{2} \mathrm{~d} x+\frac{c}{\varepsilon_{3}} \int_{0}^{1} \phi^{2} \mathrm{~d} x+\frac{c}{\varepsilon_{3}} \int_{0}^{1} \theta_{t x}^{2} \mathrm{~d} x+\frac{\delta b}{\rho J} \int_{0}^{1} \phi_{x}^{2} \mathrm{~d} x .
\end{aligned}
$$

Thus, by taking $\varepsilon_{3}$ small enough such that

$$
m_{0}=\left(\frac{b \mu}{\rho J}-\varepsilon_{3}\right)>0,
$$

we obtain (4.12).

Lemma 4.5 Let $(u, \phi, \theta)$ be the solution of (2.1)-(2.3). Then the functional

$$
F_{4}(t):=-\rho \int_{0}^{1} u_{t} u \mathrm{~d} x
$$

satisfies

$$
F_{4}^{\prime}(t) \leq-\rho \int_{0}^{1} u_{t}^{2} \mathrm{~d} x+\frac{3 \mu}{2} \int_{0}^{1} u_{x}^{2} \mathrm{~d} x+c \int_{0}^{1} \phi_{x}^{2} \mathrm{~d} x .
$$

Proof A differentiation of $F_{4}$, using (2.1) and integrating by parts and (2.2), gives

$$
\begin{aligned}
F_{4}^{\prime}(t) & =-\rho \int_{0}^{1} u_{t t} u \mathrm{~d} x-\rho \int_{0}^{1} u_{t}^{2} \mathrm{~d} x \\
& =-\mu \int_{0}^{1} u_{x x} u \mathrm{~d} x-b \int_{0}^{1} \phi_{x} u \mathrm{~d} x-\rho \int_{0}^{1} u_{t}^{2} \mathrm{~d} x \\
& =\mu \int_{0}^{1} u_{x}^{2} \mathrm{~d} x+b \int_{0}^{1} \phi u_{x} \mathrm{~d} x-\rho \int_{0}^{1} u_{t}^{2} \mathrm{~d} x .
\end{aligned}
$$

Then use of Young's and Poincaré's inequalities leads to

$$
\begin{aligned}
F_{4}^{\prime}(t) & \leq-\rho \int_{0}^{1} u_{t}^{2} \mathrm{~d} x+\mu \int_{0}^{1} u_{x}^{2} \mathrm{~d} x+\frac{\mu}{2} \int_{0}^{1} u_{x}^{2} \mathrm{~d} x+\frac{b}{2 \mu} \int_{0}^{1} \phi^{2} \mathrm{~d} x \\
& \leq-\rho \int_{0}^{1} u_{t}^{2} \mathrm{~d} x+\frac{3 \mu}{2} \int_{0}^{1} u_{x}^{2} \mathrm{~d} x+c \int_{0}^{1} \phi_{x}^{2} \mathrm{~d} x .
\end{aligned}
$$

Lemma 4.6 Let $(u, \phi, \theta)$ be the solution of (2.1)-(2.3). Then the functional

$$
F_{5}(t):=\alpha \int_{0}^{1} \theta \theta_{t} \mathrm{~d} x+\frac{k}{2} \int_{0}^{1} \theta_{x}^{2} \mathrm{~d} x+\beta \int_{0}^{1} \phi_{x} \theta \mathrm{d} x
$$

satisfies, for $\varepsilon_{2}>0$,

$$
F_{5}^{\prime}(t) \leq-\delta \int_{0}^{1} \theta_{x}^{2} \mathrm{~d} x+\varepsilon_{2} \int_{0}^{1} \phi_{x}^{2} \mathrm{~d} x+\left(\alpha+\frac{\beta^{2}}{4 \varepsilon_{2}}\right) \int_{0}^{1} \theta_{t}^{2} \mathrm{~d} x .
$$

I Springer 
Proof A simple differentiation of $F_{5}$, using (2.1), (2.2) and integrating by parts, leads to

$$
\begin{aligned}
F_{5}^{\prime}(t)= & \alpha \int_{0}^{1} \theta_{t}^{2} \mathrm{~d} x+\alpha \int_{0}^{1} \theta \theta_{t t} \mathrm{~d} x+k \int_{0}^{1} \theta_{t x} \theta_{x} \mathrm{~d} x+\beta \int_{0}^{1} \phi_{t x} \theta \mathrm{d} x+\beta \int_{0}^{1} \phi_{x} \theta_{t} \mathrm{~d} x \\
= & \alpha \int_{0}^{1} \theta_{t}^{2} \mathrm{~d} x+\delta \int_{0}^{1} \theta_{x x} \theta \mathrm{d} x-\beta \int_{0}^{1} \phi_{t x} \theta \mathrm{d} x+k \int_{0}^{1} \theta_{t x x} \theta \mathrm{d} x+k \int_{0}^{1} \theta_{t x} \theta_{x} \mathrm{~d} x \\
& +\beta \int_{0}^{1} \phi_{t x} \theta \mathrm{d} x+\beta \int_{0}^{1} \phi_{x} \theta_{t} \mathrm{~d} x \\
= & \alpha \int_{0}^{1} \theta_{t}^{2} \mathrm{~d} x-\delta \int_{0}^{1} \theta_{x}^{2} \mathrm{~d} x+\beta \int_{0}^{1} \phi_{x} \theta_{t} \mathrm{~d} x
\end{aligned}
$$

Next, by Young's inequality, we arrive at

$$
\begin{aligned}
F_{5}^{\prime}(t) & \leq-\delta \int_{0}^{1} \theta_{x}^{2} \mathrm{~d} x+\alpha \int_{0}^{1} \theta_{t}^{2} \mathrm{~d} x+\varepsilon_{2} \int_{0}^{1} \phi_{x}^{2} \mathrm{~d} x+\frac{\beta^{2}}{4 \varepsilon_{2}} \int_{0}^{1} \theta_{t}^{2} \mathrm{~d} x \\
& \leq-\delta \int_{0}^{1} \theta_{x}^{2} \mathrm{~d} x+\varepsilon_{2} \int_{0}^{1} \phi_{x}^{2} \mathrm{~d} x+\left(\alpha+\frac{\beta^{2}}{4 \varepsilon_{2}}\right) \int_{0}^{1} \theta_{t}^{2} \mathrm{~d} x .
\end{aligned}
$$

Lemma 4.7 Let $(u, \phi, \theta)$ be the solution of (2.1)-(2.3). Then, for $N, N_{1}, N_{2}, N_{3}, N_{5}>0$, to be chosen properly, the Lyapunov functional, defined by

$$
\mathcal{L}(t):=N E(t)+N_{1} F_{1}(t)+N_{2} F_{2}(t)+N_{3} F_{3}(t)+F_{4}(t)+N_{5} F_{5}(t),
$$

satisfies, for $N$ sufficiently large,

$$
\mathcal{L} \sim E
$$

and the estimate

$$
\mathcal{L}^{\prime}(t) \leq-\lambda \int_{0}^{1}\left(u_{t}^{2}+\phi_{t}^{2}+\theta_{t}^{2}+u_{x}^{2}+\phi_{x}^{2}+\theta_{x}^{2}+\phi^{2}\right) \mathrm{d} x,
$$

where $\lambda$ is a positive constant.

Proof The equivalence (4.19) is a matter of a routine calculations. See, for instance [5].

To prove (4.20), we differentiate $\mathcal{L}(t)$, and recall (4.2), (4.5), (4.10), (4.12), (4.15) and (4.17). So, we have

$$
\begin{aligned}
\mathcal{L}^{\prime}(t) \leq & -N k \int_{0}^{1} \theta_{t x}^{2} \mathrm{~d} x-\frac{N_{1} \delta}{2} \int_{0}^{1} \phi_{x}^{2} \mathrm{~d} x-N_{1}\left(\xi-\frac{b^{2}}{\mu}\right) \int_{0}^{1} \phi^{2} \mathrm{~d} x+N_{1} \varepsilon_{1} \int_{0}^{1} u_{t}^{2} \mathrm{~d} x+N_{1} c \int_{0}^{1} \theta_{t}^{2} \mathrm{~d} x \\
& +N_{1}\left(J+\frac{c}{\varepsilon_{1}}\right) \int_{0}^{1} \phi_{t}^{2} \mathrm{~d} x-N_{2} \frac{\beta}{2} \int_{0}^{1} \phi_{t}^{2} \mathrm{~d} x+N_{2} \varepsilon_{2} c \int_{0}^{1}\left(\phi_{x}^{2}+u_{x}^{2}\right) \mathrm{d} x \\
& +N_{2} c\left(1+\frac{1}{\varepsilon_{2}}\right) \int_{0}^{1} \theta_{t x}^{2} \mathrm{~d} x+\frac{\delta^{2}}{\beta} N_{2} \int_{0}^{1} \theta_{x}^{2} \mathrm{~d} x-N_{3} m_{0} \int_{0}^{1} u_{x}^{2} \mathrm{~d} x+N_{3} c \int_{0}^{1} \phi^{2} \mathrm{~d} x \\
& +N_{3} c \int_{0}^{1} \theta_{t x}^{2} \mathrm{~d} x+N_{3} \frac{\delta b}{\rho J} \int_{0}^{1} \phi_{x}^{2} \mathrm{~d} x-\rho \int_{0}^{1} u_{t}^{2} \mathrm{~d} x+\frac{3 \mu}{2} \int_{0}^{1} u_{x}^{2} \mathrm{~d} x \\
& +c \int_{0}^{1} \phi_{x}^{2} \mathrm{~d} x-\delta N_{5} \int_{0}^{1} \theta_{x}^{2} \mathrm{~d} x+N_{5} \varepsilon_{2} \int_{0}^{1} \phi_{x}^{2} \mathrm{~d} x+\left(\alpha+\frac{\beta^{2}}{4 \varepsilon_{2}}\right) N_{5} \int_{0}^{1} \theta_{t}^{2} \mathrm{~d} x .
\end{aligned}
$$

We apply Poincaré's inequality for $\theta_{t}$ and take $N_{5}=\frac{2 \delta}{\beta} N_{2}$, to get

$$
\begin{aligned}
\mathcal{L}^{\prime}(t) \leq & -\left[N k-N_{1} c-N_{2} c\left(1+\frac{1}{\varepsilon_{2}}\right)-N_{3} c\right] \int_{0}^{1} \theta_{t x}^{2} \mathrm{~d} x \\
& -\left[\frac{N_{1} \delta}{2}-N_{2} c \varepsilon_{2}-N_{3} \frac{\delta b}{\rho J}-c\right] \int_{0}^{1} \phi_{x}^{2} \mathrm{~d} x-\left[N_{1}\left(\xi-\frac{b^{2}}{\mu}\right)-N_{3} c\right] \int_{0}^{1} \phi^{2} \mathrm{~d} x
\end{aligned}
$$




$$
\begin{aligned}
& -\left(\rho-N_{1} \varepsilon_{1}\right) \int_{0}^{1} u_{t}^{2} \mathrm{~d} x-\left[N_{2} \frac{\beta}{2}-N_{1}\left(J+\frac{c}{\varepsilon_{1}}\right)\right] \int_{0}^{1} \phi_{t}^{2} \mathrm{~d} x-\frac{\delta^{2}}{\beta} N_{2} \int_{0}^{1} \theta_{x}^{2} \mathrm{~d} x \\
& -\left(N_{3} m_{0}-N_{2} c \varepsilon_{2}-\frac{3 \mu}{2}\right) \int_{0}^{1} u_{x}^{2} \mathrm{~d} x .
\end{aligned}
$$

At this point, we choose the constants carefully. First, let us take $\varepsilon_{1}=\frac{\rho}{2 N_{1}}$, and choose $N_{3}$ large enough such that

$$
\alpha_{1}=N_{3} m_{0}-\frac{3 \mu}{2}>0
$$

We then choose $N_{1}$ large enough so that

$$
\alpha_{2}=N_{1}\left(\xi-\frac{b^{2}}{\mu}\right)-N_{3} c>0, \alpha_{3}=N_{1} \frac{\delta}{2}-\left(N_{3} \frac{\delta b}{J \rho}+c\right)>0 .
$$

Next, we select $N_{2}$ so large that

$$
\alpha_{4}=N_{2} \frac{\beta}{2}-N_{1}\left(J+\frac{2 c N_{1}}{\rho}\right)>0,
$$

then pick $\varepsilon_{2}$ small enough so that

$$
\alpha_{5}=\alpha_{1}-N_{2} c \varepsilon_{2}>0, \quad \alpha_{6}=\alpha_{3}-N_{2} c \varepsilon_{2}>0 .
$$

Finally, we choose $N$ large enough so that (4.19) remains valid and, further,

$$
\alpha_{7}=N k-N_{1} c-N_{2} c\left(1+\frac{1}{\varepsilon_{2}}\right)-N_{3} c>0 .
$$

Therefore, we arrive at

$$
\begin{aligned}
\mathcal{L}^{\prime}(t) \leq & -\alpha_{7} \int_{0}^{1} \theta_{t x}^{2} \mathrm{~d} x-\alpha_{6} \int_{0}^{1} \phi_{x}^{2} \mathrm{~d} x-\alpha_{2} \int_{0}^{1} \phi^{2} \mathrm{~d} x-\frac{\rho}{2} \int_{0}^{1} u_{t}^{2} \mathrm{~d} x \\
& -\alpha_{4} \int_{0}^{1} \phi_{t}^{2} \mathrm{~d} x-\alpha_{5} \int_{0}^{1} u_{x}^{2} \mathrm{~d} x-c \int_{0}^{1} \theta_{x}^{2} \mathrm{~d} x .
\end{aligned}
$$

We finally use Poincaré's inequality to substitute $-\int_{0}^{1} \theta_{x t}^{2} \mathrm{~d} x$ by $-\int_{0}^{1} \theta_{t}^{2} \mathrm{~d} x$ and, hence, (4.20) is established.

Theorem 4.8 Let $(u, \phi, \theta)$ be the solution of (2.1)-(2.3) and assume (1.7). Then there exist two positive constants $k_{1}$ and $k_{2}$ such that the energy functional (4.1) satisfies

$$
E(t) \leq k_{1} e^{-k_{2} t}, \quad \forall t \geq 0 .
$$

Proof First, using Young's inequality, (4.1) becomes

$$
E(t) \leq c \int_{0}^{1}\left[u_{t}^{2}+\phi_{t}^{2}+\theta_{t}^{2}+u_{x}^{2}+\phi_{x}^{2}+\theta_{x}^{2}+\phi^{2}\right] \mathrm{d} x .
$$

The combination of (4.20) and (4.22) gives

$$
\mathcal{L}^{\prime}(t) \leq-c E(t), \quad \forall t \geq 0 .
$$

Using $\mathcal{L} \sim E$, we get

$$
\mathcal{L}^{\prime}(t) \leq-k_{2} \mathcal{L}(t), \quad \forall t \geq 0 .
$$

A simple integration over $(0, t)$ yields

$$
\mathcal{L}(t) \leq \mathcal{L}(0) e^{-k_{2} t}, \quad \forall t \geq 0 .
$$

Consequently, (4.21) is established by recalling $\mathcal{L} \sim E$. 


\section{Polynomial stability}

In this section, we prove the polynomial decay result for the non-equal speed of propagation case, that is (1.7) does not holds. To establish our result, we work with the strong solution of (2.1)-(2.3) and define the second-order energy functional

$$
E_{2}(t)=\frac{1}{2} \int_{0}^{1}\left[\rho u_{t t}^{2}+J \phi_{t t}^{2}+\alpha \theta_{t t}^{2}+\mu u_{t x}^{2}+\delta \phi_{t x}^{2}+\delta \theta_{t x}^{2}+2 b u_{t x} \phi_{t}+\xi \phi_{t}^{2}\right] \mathrm{d} x .
$$

Similar calculations, as in Lemma 4.1, lead to

$$
E_{2}^{\prime}(t)=-k \int_{0}^{1} \theta_{t t x}^{2} \mathrm{~d} x \leq 0
$$

Lemma 5.1 Let $(u, \phi, \theta)$ be the strong solution of (2.1)-(2.3). Then the functional

$$
\tilde{F}_{3}(t):=\beta F_{3}(t)-\chi k \int_{0}^{1} u_{x} \theta_{t x} \mathrm{~d} x-\chi \delta \int_{0}^{1} u_{x} \theta_{x} \mathrm{~d} x
$$

satisfies, for any $\varepsilon_{7}>0$ and for some positive constant $m_{1}$, the estimate

$$
\tilde{F}_{3}^{\prime}(t) \leq-m_{1} \int_{0}^{1} u_{x}^{2} \mathrm{~d} x+c \int_{0}^{1} \phi^{2} \mathrm{~d} x+c_{2} \int_{0}^{1} \theta_{t x}^{2} \mathrm{~d} x+\frac{\delta \beta b}{\rho J} \int_{0}^{1} \phi_{x}^{2} \mathrm{~d} x+c_{7} \int_{0}^{1} \theta_{t t x}^{2} \mathrm{~d} x+\varepsilon_{7} \int_{0}^{1} u_{t}^{2} \mathrm{~d} x .
$$

Proof A simple differentiation of (5.3) gives

$$
\tilde{F}_{3}^{\prime}(t)=\beta F_{3}^{\prime}(t)-\chi k \int_{0}^{1} u_{t x} \theta_{t x} \mathrm{~d} x-\chi k \int_{0}^{1} u_{x} \theta_{t t x} \mathrm{~d} x-\chi \delta \int_{0}^{1} u_{t x} \theta_{x} \mathrm{~d} x-\chi \delta \int_{0}^{1} u_{x} \theta_{t x} \mathrm{~d} x .
$$

Using integration by parts for the second term in the right-hand of (5.5) and exploiting $(2.1)_{3}$, we get

$$
-\chi k \int_{0}^{1} u_{t x} \theta_{t x} \mathrm{~d} x=\chi k \int_{0}^{1} u_{t} \theta_{t x x} \mathrm{~d} x=\alpha \chi \int_{0}^{1} u_{t} \theta_{t t} \mathrm{~d} x-\delta \chi \int_{0}^{1} u_{t} \theta_{x x} \mathrm{~d} x+\beta \chi \int_{0}^{1} u_{t} \phi_{t x} \mathrm{~d} x .
$$

Substituting (5.6) and (4.13) into (5.5), we obtain

$$
\begin{aligned}
\tilde{F}_{3}^{\prime}(t)= & \frac{-b \beta \mu}{\rho J} \int_{0}^{1} u_{x}^{2} \mathrm{~d} x-\frac{\mu \beta \xi}{\rho J} \int_{0}^{1} \phi u_{x} \mathrm{~d} x-\frac{\beta^{2} \mu}{\rho J} \int_{0}^{1} \theta_{t x} u_{x} \mathrm{~d} x+\frac{\delta \beta b}{\rho J} \int_{0}^{1} \phi_{x}^{2} \mathrm{~d} x+\alpha \chi \int_{0}^{1} u_{t} \theta_{t t} \mathrm{~d} x \\
& -\chi k \int_{0}^{1} u_{x} \theta_{t t x} \mathrm{~d} x-\chi \delta \int_{0}^{1} u_{x} \theta_{t x} \mathrm{~d} x .
\end{aligned}
$$

Using Young's and Poincaré's inequalities, we find

$$
\begin{aligned}
\tilde{F}_{3}^{\prime}(t) \leq & -\left(\frac{b \beta \mu}{\rho J}-\varepsilon_{6}\right) \int_{0}^{1} u_{x}^{2} \mathrm{~d} x+\frac{c}{\varepsilon_{6}} \int_{0}^{1} \phi^{2} \mathrm{~d} x+\frac{c}{\varepsilon_{6}} \int_{0}^{1} \theta_{t x}^{2} \mathrm{~d} x+\frac{\delta \beta b}{\rho J} \int_{0}^{1} \phi_{x}^{2} \mathrm{~d} x+\varepsilon_{7} \int_{0}^{1} u_{t}^{2} \mathrm{~d} x \\
& +c\left(\frac{1}{\varepsilon_{6}}+\frac{1}{\varepsilon_{7}}\right) \int_{0}^{1} \theta_{t t x}^{2} \mathrm{~d} x .
\end{aligned}
$$

Finally, we choose $\varepsilon_{6}$ small enough such that

$$
m_{1}=\frac{b \beta \mu}{\rho J}-\varepsilon_{6}>0,
$$

to obtain (5.4). 
Lemma 5.2 Let $(u, \phi, \theta)$ be the strong solution of (2.1)-(2.3). Then the Lyapunov functional defined by

$$
\tilde{\mathcal{L}}(t):=N^{*}\left(E(t)+E_{2}(t)\right)+N_{1}^{*} F_{1}(t)+N_{2}^{*} F_{2}(t)+N_{3}^{*} \tilde{F}_{3}(t)+F_{4}(t)+N_{5}^{*} F_{5}(t)
$$

satisfies, for $N^{*}, N_{1}^{*}, N_{2}^{*}, N_{3}^{*}, N_{5}^{*}>0$ to be chosen properly, and for a positive constant $\lambda_{1}$,

$$
\tilde{\mathcal{L}}^{\prime}(t) \leq-\lambda_{1} \int_{0}^{1}\left(u_{t}^{2}+\phi_{t}^{2}+\theta_{t}^{2}+u_{x}^{2}+\phi_{x}^{2}+\theta_{x}^{2}+\phi^{2}\right) \mathrm{d} x .
$$

Proof By exploiting (5.4) and the fact $\mu \xi>b^{2}$ we get

$$
\begin{aligned}
\tilde{\mathcal{L}}^{\prime}(t) \leq & -N^{*} k \int_{0}^{1} \theta_{t x}^{2} \mathrm{~d} x-N^{*} k \int_{0}^{1} \theta_{t t x}^{2} \mathrm{~d} x-\frac{N_{1}^{*} \delta}{2} \int_{0}^{1} \phi_{x}^{2} \mathrm{~d} x-N_{1}^{*}\left(\xi-\frac{b^{2}}{\mu}\right) \int_{0}^{1} \phi^{2} \mathrm{~d} x \\
& +N_{1}^{*} \varepsilon_{1} \int_{0}^{1} u_{t}^{2} \mathrm{~d} x+N_{1}^{*} c \int_{0}^{1} \theta_{t}^{2} \mathrm{~d} x+N_{1}^{*}\left(J+\frac{c}{\varepsilon_{1}}\right) \int_{0}^{1} \phi_{t}^{2} \mathrm{~d} x-N_{2}^{*} \frac{\beta}{2} \int_{0}^{1} \phi_{t}^{2} \mathrm{~d} x+N_{2}^{*} \frac{\delta^{2}}{\beta} \int_{0}^{1} \theta_{x}^{2} \mathrm{~d} x \\
& +N_{2}^{*} c \varepsilon_{2} \int_{0}^{1}\left(\phi_{x}^{2}+u_{x}^{2}\right) \mathrm{d} x+N_{2}^{*} c\left(1+\frac{1}{\varepsilon_{2}}\right) \int_{0}^{1} \theta_{t x}^{2} \mathrm{~d} x-N_{3}^{*} m_{1} \int_{0}^{1} u_{x}^{2} \mathrm{~d} x+N_{3}^{*} c \int_{0}^{1} \phi^{2} \mathrm{~d} x \\
& +N_{3}^{*} c_{2} \int_{0}^{1} \theta_{t x}^{2} \mathrm{~d} x+N_{3}^{*} \frac{\delta b \beta}{\rho J} \int_{0}^{1} \phi_{x}^{2} \mathrm{~d} x+N_{3}^{*} c_{3} \int_{0}^{1} \theta_{t t x}^{2} \mathrm{~d} x+N_{3}^{*} \varepsilon_{7} \int_{0}^{1} u_{t}^{2} \mathrm{~d} x-\rho \int_{0}^{1} u_{t}^{2} \mathrm{~d} x \\
& +\frac{3 \mu}{2} \int_{0}^{1} u_{x}^{2} \mathrm{~d} x+c \int_{0}^{1} \phi_{x}^{2} \mathrm{~d} x-N_{5}^{*} \delta \int_{0}^{1} \theta_{x}^{2} \mathrm{~d} x+N_{5}^{*} \varepsilon_{2} \int_{0}^{1} \phi_{x}^{2} \mathrm{~d} x+N_{5}^{*}\left(\alpha+\frac{\beta^{2}}{4 \varepsilon_{2}}\right) \int_{0}^{1} \theta_{t}^{2} \mathrm{~d} x .
\end{aligned}
$$

We apply Poincaré's inequality for $\theta_{t}$ to get

$$
\begin{aligned}
\tilde{\mathcal{L}}^{\prime}(t) \leq & -\left[N^{*} k-N_{1}^{*} c-N_{2}^{*} c\left(1+\frac{1}{\varepsilon_{2}}\right)-N_{3}^{*} c_{2}-N_{5}^{*} c\left(1+\frac{1}{\varepsilon_{2}}\right)\right] \int_{0}^{1} \theta_{t x}^{2} \mathrm{~d} x \\
& -\left[\frac{N_{1}^{*} \delta}{2}-N_{2}^{*} c \varepsilon_{2}-N_{3}^{*} \frac{\delta \beta b}{\rho J}-c-N_{5}^{*} \varepsilon_{2}\right] \int_{0}^{1} \phi_{x}^{2} \mathrm{~d} x-\left[N_{1}^{*}\left(\xi-\frac{b^{2}}{\mu}\right)-N_{3}^{*} c\right] \int_{0}^{1} \phi^{2} \mathrm{~d} x \\
& -\left(\rho-N_{1}^{*} \varepsilon_{1}-N_{3}^{*} \varepsilon_{7}\right) \int_{0}^{1} u_{t}^{2} \mathrm{~d} x-\left[N_{2}^{*} \frac{\beta}{2}-N_{1}^{*}\left(J+\frac{c}{\varepsilon_{1}}\right)\right] \int_{0}^{1} \phi_{t}^{2} \mathrm{~d} x-\left(\delta N_{5}^{*}-\frac{\delta^{2}}{\beta} N_{2}^{*}\right) \int_{0}^{1} \theta_{x}^{2} \mathrm{~d} x \\
& -\left(N_{3}^{*} m_{1}-N_{2}^{*} c \varepsilon_{2}-\frac{3 \mu}{2}\right) \int_{0}^{1} u_{x}^{2} \mathrm{~d} x-\left(N^{*} k-N_{3}^{*} c_{3}\right) \int_{0}^{1} \theta_{t t x}^{2} \mathrm{~d} x .
\end{aligned}
$$

Similarly to what we did with $\mathcal{L}^{\prime}$, we take $\varepsilon_{1}=\frac{\rho}{4 N_{1}^{*}}, N_{5}^{*}=\frac{2 \delta}{\beta} N_{2}^{*}$ and $\varepsilon_{7}=\frac{\rho}{4 N_{3}^{*}}$ and then choose $N_{3}^{*}$ large enough such that

$$
\alpha_{1}^{*}=N_{3}^{*} m_{1}-\frac{3 \mu}{2}>0
$$

and select $N_{1}^{*}$ large enough so that

$$
\alpha_{2}^{*}=N_{1}^{*}\left(\xi-\frac{b^{2}}{\mu}\right)-N_{3}^{*} c>0
$$

and

$$
\alpha_{3}^{*}=N_{1}^{*} \frac{\delta}{2}-\left(N_{3}^{*} \frac{\delta b \beta}{J \rho}+c\right)>0 .
$$

Next we choose $N_{2}^{*}$ so large that

$$
\alpha_{4}^{*}=N_{2}^{*} \frac{\beta}{2}-N_{1}^{*}\left(J+\frac{4 c N_{1}}{\rho}\right)>0,
$$


then pick $\varepsilon_{2}$ small enough such that

$$
\alpha_{5}^{*}=\alpha_{1}^{*}-N_{2}^{*} c \varepsilon_{2}>0
$$

and

$$
\alpha_{6}^{*}=\alpha_{3}^{*}-N_{2}^{*} c \varepsilon_{2}>0 .
$$

Finally, we take $N^{*}$ large enough such that

$$
\alpha_{7}^{*}=N^{*} k-N_{1}^{*} c-N_{2}^{*} c\left(1+\frac{1}{\varepsilon_{2}}\right)-N_{3}^{*} c>0,
$$

and

$$
\alpha_{8}^{*}=N^{*} k-N_{3}^{*} c_{3}>0 .
$$

Therefore, using Poincaré's inequality, we arrive at

$$
\begin{aligned}
\mathcal{L}^{\prime}(t) \leq & -\alpha_{7}^{*} c \int_{0}^{1} \theta_{t}^{2} \mathrm{~d} x-\alpha_{6}^{*} \int_{0}^{1} \phi_{x}^{2} \mathrm{~d} x-\alpha_{2}^{*} \int_{0}^{1} \phi^{2} \mathrm{~d} x-\frac{\rho}{2} \int_{0}^{1} u_{t}^{2} \mathrm{~d} x-\alpha_{4}^{*} \int_{0}^{1} \phi_{t}^{2} \mathrm{~d} x-\alpha_{5}^{*} \int_{0}^{1} u_{x}^{2} \mathrm{~d} x \\
& -c \int_{0}^{1} \theta_{x}^{2} \mathrm{~d} x-\alpha_{8}^{*} \int_{0}^{1} \theta_{t t x}^{2} \mathrm{~d} x .
\end{aligned}
$$

So, there exists $\lambda_{1}>0$ such that

$$
\tilde{\mathcal{L}}^{\prime}(t) \leq-\lambda_{1} \int_{0}^{1}\left(u_{t}^{2}+\phi_{t}^{2}+\theta_{t}^{2}+u_{x}^{2}+\phi_{x}^{2}+\theta_{x}^{2}+\phi^{2}\right) \mathrm{d} x .
$$

Theorem 5.3 Let $(u, \phi, \theta)$ be the strong solution of (2.1)-(2.3) and assume that (1.7) does not hold. Then there exists a positive constant $k_{2}$, independent of $t$ and the initial data, such that

$$
E(t) \leq \frac{k_{2}\left(E(0)+E_{2}(0)\right)}{t}, \forall t>0
$$

Proof The combination of (4.22) and (5.8) gives

$$
\tilde{\mathcal{L}}^{\prime}(t) \leq-\frac{\lambda_{1}}{c} E(t), \quad \forall t>0 .
$$

We integrate the last inequality over $(0, t)$, and recall that $\tilde{\mathcal{L}}^{\prime}$ is non-increasing, we obtain

$$
\begin{aligned}
& \int_{0}^{t} E(s) \mathrm{d} s \leq-\frac{c}{\lambda_{1}} \int_{0}^{t} \tilde{\mathcal{L}}^{\prime}(s) \mathrm{d} s, \\
& \int_{0}^{t} E(s) \mathrm{d} s \leq \frac{c}{\lambda_{1}} \tilde{\mathcal{L}}(0), \quad \forall t>0 .
\end{aligned}
$$

Using the fact $t E(t) \leq \int_{0}^{t} E(s) \mathrm{d} s$, we find

$$
E(t) \leq \lambda_{2} \frac{\tilde{\mathcal{L}}(0)}{t}, \quad \forall t>0 .
$$

Consequently, there exists $k_{2}$ positive such that

$$
E(t) \leq \frac{k_{2}\left(E(0)+E_{2}(0)\right)}{t}, \forall t>0 .
$$

This finishes the proof.

Remark 5.4 We note here that these results hold even for $\mu \xi=b^{2}$. In this case, we have to redefine the energy as in [10] and adjust our calculations accordingly. In particular, when $\mu=\xi=b$, our system reduces to Timoshenko system with thermoelasticity type III. This has been discussed and similar stability results have been established in [20,21]. 


\section{Numerical tests}

To illustrate the theoretical results of this work, we present in this section two numerical tests. We solve the system (2.1) under the initial and boundary conditions (2.2), (2.3). The system is discritized using a second order finite difference method in time and space. For more stability, we implement the conservative scheme of Lax-Wendroff. for more details, we refer to our previous works $[1,11,17]$. We examine the following two tests:

- TEST 1: Based on the result (4.21) of our Theorem, we examine the exponential decay of the energy (4.1) using the equality condition of the parameters $\chi=0$, given by (1.7). Here, we take all parameters of the system (2.1) equal to 1.

- TEST 2: In Test 2, we examine the polynomial decay of the energy (4.1) using the parameters condition $\chi \neq 0$, where the parameters of the system (2.1) are taken as follows $\mu=5 ; \rho=1 ; \delta=0.05 ; J=1$ and the remaining parameters are equal to 1 .

To ensure the numerical stability of the implemented method and the executed code, we use $\Delta t<<$ $0.5 \mathrm{~d} x$ satisfying the stability condition according to the Courant-Friedrichs-Lewy (CFL) inequality, where $d t$ represents the time step and $\mathrm{d} x$ the spatial step. The spatial interval $[0,1]$ is subdivided into 200 subintervals and the temporal interval $\left[0, T_{e}\right]=[0,1]$ is deduced from the stability condition above. We run our code for 10,000 time steps using the following initial conditions:

$$
u(x, 0)=2 \sin (\pi x) ; \phi(x, 0)=2 x \sin (\pi x) ; \theta(x, 0)=\frac{1}{4} x(1-x) \quad \text { in }[0,1] .
$$

Under the same initial and boundary conditions mentioned above, we show in Fig. 1 the numerical results of the exponential decay case. Whereas we present in Fig. 2 the results obtained for the polynomial case. We show three cross section cuts for the numerical solution $(u, \phi, \theta)$ at $x=0.25, x=0.5$ and at $x=0.75$. For all components of the solution, the decay behavior is clearly demonstrated for both experiments, the exponential and the polynomial decays. Moreover, it should be stressed that the graphical presentations are normalized to ensure a clear comparisons. Therefore, we can clearly compare the energy decay obtained in Test 1 and in Test 2. For this, see Fig. 3.

Finally, we noticed that the case $\chi=0$ ensures an exponential energy decay and, therefore, the decay of all components of the solution $(u, \phi, \theta)$. While the case $\chi \neq 0$ ensures the polynomial decay. But for some special choices of the system parameters generating the damping speed, we could obtain an exponential-like decay of the energy and a damped waves similar to the exponential case.
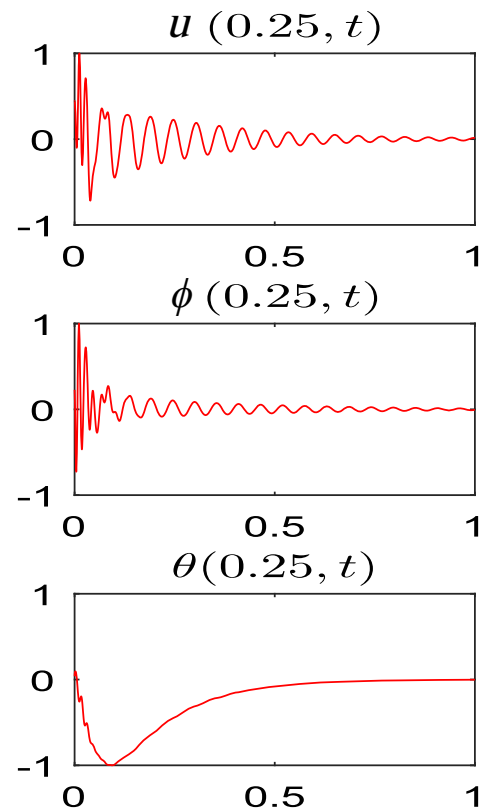
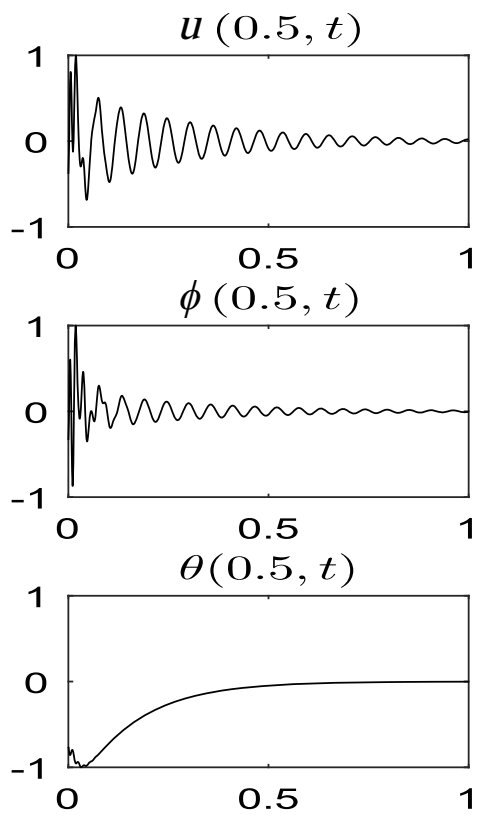
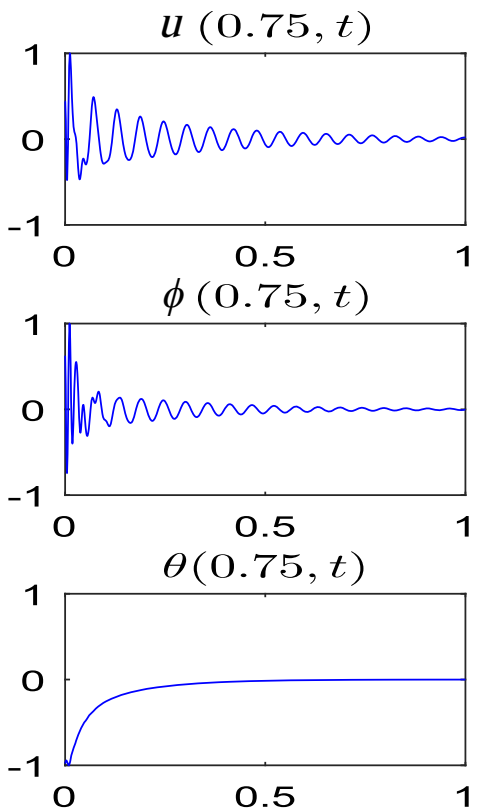

Fig. 1 TEST 1: cross section cuts of the solution for the exponential decay 

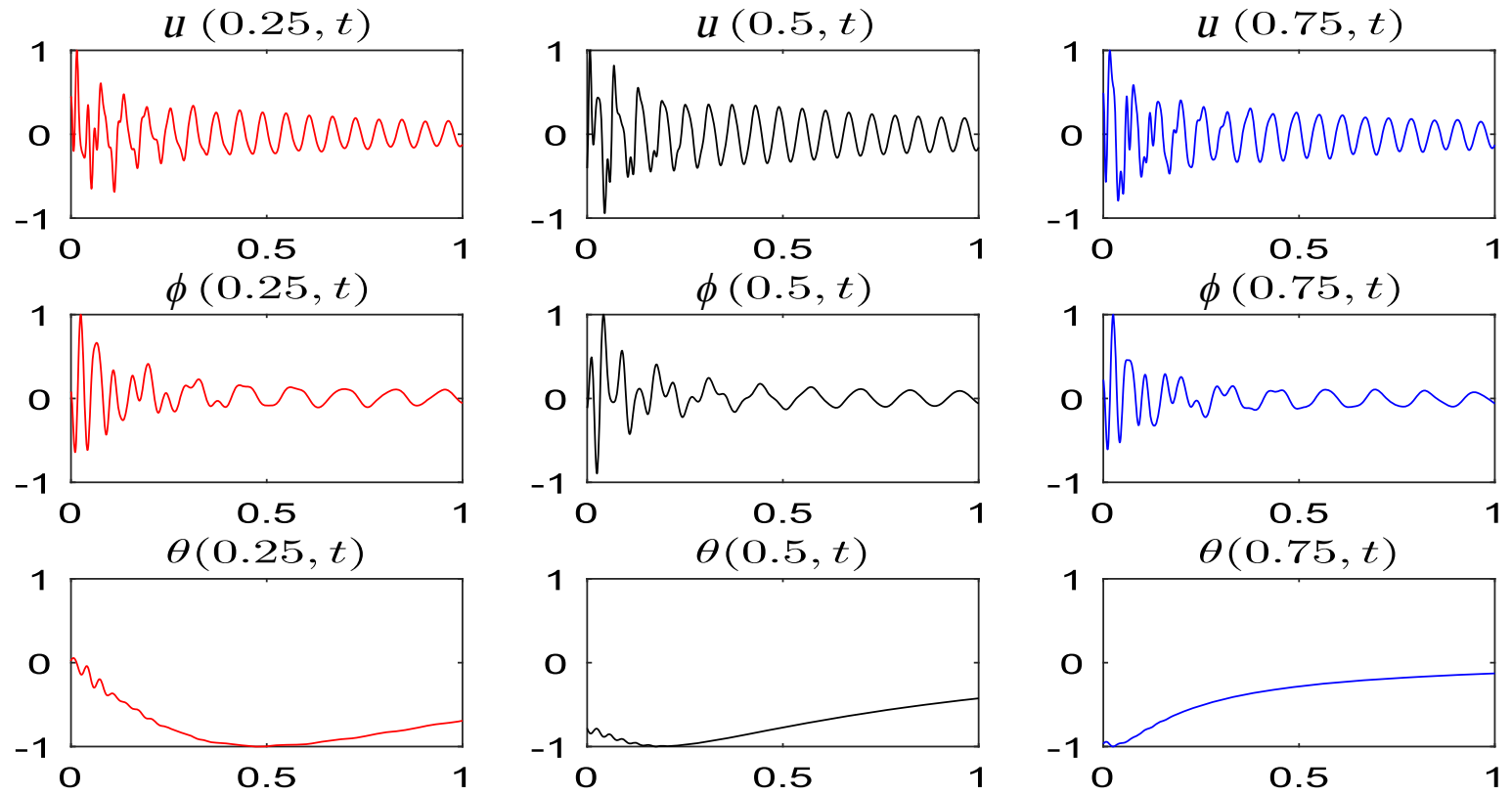

Fig. 2 TEST 2: cross section cuts of the solution for the polynomial decay

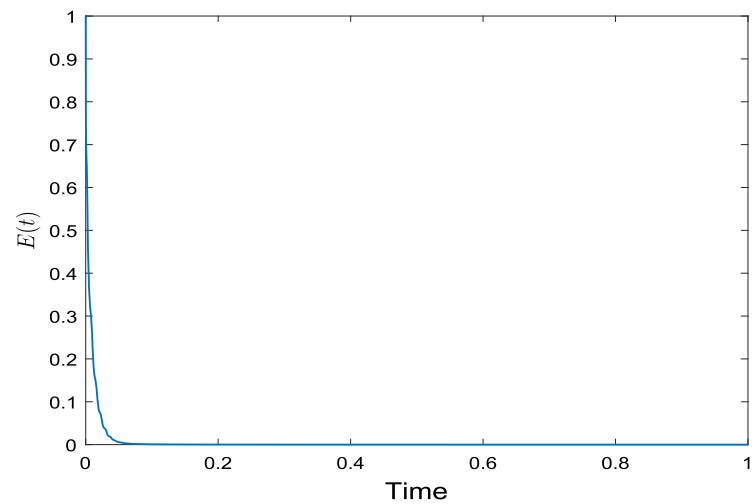

A TEST 1: Exponential decay

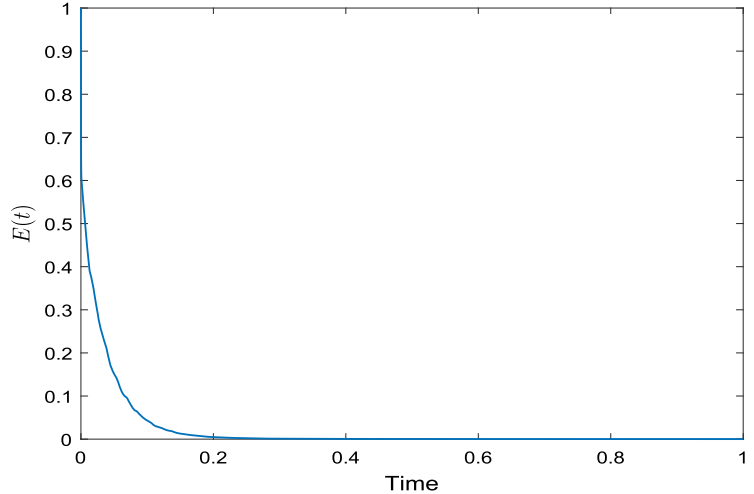

B TEST 2: Polynomial decay

Fig. 3 Energy function for the exponential and polynomial decays

Acknowledgements The authors thank very much both reviewers for their careful reading and valuable suggestions. This work was supported by MASEP Research Group in the Research Institute of Sciences and Engineering at the University of Sharjah. Grant no. 2002144089, 2019-2020.

Open Access This article is licensed under a Creative Commons Attribution 4.0 International License, which permits use, sharing, adaptation, distribution and reproduction in any medium or format, as long as you give appropriate credit to the original author(s) and the source, provide a link to the Creative Commons licence, and indicate if changes were made. The images or other third party material in this article are included in the article's Creative Commons licence, unless indicated otherwise in a credit line to the material. If material is not included in the article's Creative Commons licence and your intended use is not permitted by statutory regulation or exceeds the permitted use, you will need to obtain permission directly from the copyright holder. To view a copy of this licence, visit http://creativecommons.org/licenses/by/4.0/.

\section{References}

1. Afilal, M.; Guesmia, A.; Soufyane, A.; Zahri, M.: On the exponential and polynomial stability for a linear Bresse system. Math. Methods Appl. Sci. 43(5), 2626-2645 (2020)

2. Apalara, T.A.: Exponential decay in one-dimensional porous dissipation elasticity. Q. J. Mech. Appl. Math. 70, 363-372 (2017) 
3. Apalara, T.A.: Corrigendum: exponential decay in one-dimensional porous dissipation elasticity. Q. J. Mech. Appl. Math. 70(4), 553-555 (2017)

4. Apalara, T.A.: On the stability of porous-elastic system with microtemparatures. J. Therm. Stress. 42(2), 265-278 (2019)

5. Apalara, T.A.: A general decay for a weakly nonlinearly damped porous system. J. Dyn. Control Syst. 25(3), 311-322 (2019)

6. Chandrasekharaiah, D.S.: Hyperbolic thermoelasticity: a review of recent literature. Appl. Mech. Rev. 51(12), 705-729 (1998)

7. Chandrasekharaiah, D.S.: Thermoelasticity with thermal relaxation: an alternative formulation. Proc. Indian Acad. Sci. (Math Sci). 109(1), 95-106 (1999)

8. Cowin, S.C.: The viscoelastic behavior of linear elastic materials with voids. J. Elast. 15, 185-191 (1985)

9. Cowin, S.C.; Nunziato, J.W.: Linear elastic materials with voids. J. Elast. 13, 125-147 (1983)

10. Fareh, A.; Messaoudi, S.A.: Energy decay for a porous thermoelastic system with thermoelasticity of second sound and with a non-necessary positive definite energy. Appl. Math. Comput. 293, 493-507 (2017)

11. Feng, B.; Zahri, M.: Optimal decay rate estimates of a nonlinear viscoelastic Kirchhoff plate. Complexity 2020, 6079507 (2020)

12. Goodman, M.A.; Cowin, S.C.: A continuum theory for granular materials. Arch. Ration. Mech. Anal. 44, 249-266 (1972)

13. Green, A.E.; Naghdi, P.M.: On thermodynamics and the nature of the second law. Proc. R. Soc. Lond. Ser. A Math. Phys. Sci. 357(1690), 253-270 (1977)

14. Green, A.E.; Naghdi, P.M.: A re-examination of the basic postulates of thermomechanics. Proc. R. Soc. Lond. Ser. A Math. Phys. Sci. 432(1885), 171-194 (1991)

15. Green, A.E.; Naghdi, P.M.: On undamped heat waves in an elastic solid. J. Therm. Stress. 15(2), 253-264 (1992)

16. Green, A.E.; Naghdi, P.M.: Thermoelasticity without energy dissipation. J. Therm. Stress. 31(3), 189-208 (1993)

17. Hassan, J.H.; Messaoudi, S.A.; Zahri, M.: Existence and new general decay results for a viscoelastic-type Timoshenko system. Zeitschrift für Analysis und ihre Anwendungen 39(2), 185-222 (2020)

18. Iesan, D.; Quintanilla, R.: Decay estimates and energy bounds for porous elastic cylinders. Z. Angew. Math. Phys. 46(2), 268-281 (1995)

19. Liu, Z.; Zheng, S.: Semigroups Associated with Dissipative Systems. Chapman Hall/CRC, Boca Raton (1999)

20. Messaoudi, S.A.; Fareh, A.: Energy decay in a Timoshenko-type system of thermoelasticity of type III with different wavepropagation speeds. Arab. J. Math. 2, 199-207 (2013)

21. Messaoudi, S.A.; Said-Houari, B.: Energy decay in a Timoshenko-type system of thermoelasticity of type III. JAMA 348, 298-307 (2008)

22. Nunziato, J.W.; Cowin, S.C.: A nonlinear theory of elastic materials with voids. Arch. Ration. Mech. Anal. 72, 175-201 (1979)

23. Pazy, A.: Semigroups of Linear Operators and Applications to Partial Differential Equations. Springer, New York (1983)

24. Quintanilla, R.: Uniqueness in nonlinear theory of porous elastic materials. Arch. Mech. 49, 67-75 (1997)

25. Quintanilla, R.: Slow decay for one-dimensional porous dissipation elasticity. Appl. Math. Lett. 16, 487-491 (2003)

26. Santos, M.L.; Almeida Junior, D.S.: On porous-elastic system with localized damping. Z. Angew. Math. Phys. 67(3), 1-18 (2016)

27. Santos, M.L.; Campelo, A.D.S.; Almeida Junior, D.S.: On the decay rates of porous elastic systems. J. Elast. 127(1), 79-101 (2017)

28. Santos, M.L.; Campelo, A.D.S.; Almeida Junior, D.S.: Rates of decay for porous elastic system weakly dissipative. Acta Appl. Math. 151, 1-26 (2017)

Publisher's Note Springer Nature remains neutral with regard to jurisdictional claims in published maps and institutional affiliations. 